\title{
Quel avenir pour les régions périphériques?
}

\author{
André Joyal, chercheur \\ Centre de recherche en développement territorial
}

\section{INTRODUCTION}

\section{Un chercheur relié à un think tank londonien se demandait si, à cause de l'austérité, des villes comme Leeds et Manchester n'allaient pas perdre de leur importance en laissant toute la place à Londres.}

En Grande-Bretagne, quelques jours avant les élections du 7 mai dernier qui reportèrent au pouvoir le parti de David Cameron, un chercheur relié à un think tank londonien, se demandait si, à cause de l'austérité, des villes comme Leeds et Manchester n'allaient pas perdre de leur importance en laissant toute la place à Londres. Peut-on soulever une question similaire au Québec? Si la capitale nationale, et des villes comme Sherbrooke, Trois-Rivières et autres Saguenay ne semblent pas menacées par les politiques de l'administration Couillard, il peut en être autrement pour les petites villes et villages des régions comme celles du LacSaint-Jean, de l'Abitibi-Témiscamingue et de l'Estdu-Québec. Et pourtant, lors d'un des débats télévisés durant la campagne électorale, Philippe

\section{UN PEU D'HISTOIRE}

Le journal La Presse a consacré un dossier aux 《 villages dévitalisés » ${ }^{4}$ de la péninsule gaspésienne dotés parfois de noms empreints de poésie tels Grosses-Roches, Rivière-à-Claude auxquels s'ajoutent L'Ascension-de-Matapédia, Sainte-Madeleinede-la-Rivière-Madeleine, Saint-Maxime-du-MontLouis, Saint-Zénon-du-Lac-Humqui... Des municipalités, faut bien l'admettre, qui ne sont pas de nature à faire des rapprochements avec Leeds et Manchester, juste en face, outre-Atlantique. Ce dossier fut rédigé dans la foulée d'une déclaration intempestive du président du Conseil du patronat, M. Yves-Thomas Dorval, lors de sa participation en commission parlementaire. Ce dernier, en présentant son mémoire, a recommandé au gouvernement d'orienter une partie des budgets actuel-
Couillard se disait très préoccupé par le sort des travailleurs des Îles-de-la-Madeleine et de la Gaspésie ${ }^{1}$. Pour sa part, Martin Coiteux, grand argentier du gouvernement, en sa qualité de président du Conseil du trésor, lors de son passage à Tout le monde en parle ${ }^{2}$, malgré le caractère léger de cette émission, semblait être sérieux en disant oui, sans rire - que son gouvernement se préoccupait beaucoup du sort des PME en régions. Hélas, ce soir-là personne n'était en mesure de lui demander de justifier sa vision optimiste dans un contexte où l'appui financier aux Centres locaux de développement (CLD) allait être coupé de $55 \%$. Comme on le verra, cette décision en faveur de laquelle personne n'a voté, a soulevé une véritable levée de boucliers. Le mouvement Touche pas à ma région incarne cette opposition au sein des différentes régions administratives du Québec. Ayant rédigé un mémoire de maîtrise sur l'avenir de l'agriculture dans le Bas-St-Laurent/Gaspésie ${ }^{3}$ une attention particulière sera ici consacrée au coin de pays qui a vu naître la Bolduc (Mary Travers) et René Lévesque : la Gaspésie.

lement consacrés au maintien des municipalités dévitalisées vers un soutien à la relocalisation de leur population ${ }^{6}$. Il n'en fallait pas plus pour ranimer parmi la population gaspésienne les douloureux souvenirs de ces villages rayés de la carte dans le cadre des opérations du Bureau d'Aménagement de l'Est-du-Québec (BAEQ) dont on « célèbrera » en 2016, le $50^{\mathrm{e}}$ anniversaire de la publication des recommandations de cette vaste opération demeurée unique dans nos annales du développement économique.

On reconnaît trois phases dans l'évolution qu'ont connue les politiques économiques en matière de développement régional à l'échelle du Québec comme à celle de nombreux pays occidentaux. 


\section{La première phase : les années soixante}

Les enjeux politiques ayant eu préséance sur les enjeux économiques, la planification régionale fut reléguée aux oubliettes.

C'est la période marquée par les pôles de croissance à la Perroux (les industries industrialisantes) qui ne manqueront pas d'influencer les jeunes responsables du BAEQ surtout suite à la signature du célèbre accord de 1961 avec le gouvernement canadien (Aménagement régional et développement agricole: ARDA) ayant trait aux régions défavorisées de l'Est du Canada. On parlait alors du nivèlement des disparités régionales à la faveur d'une approche fonctionnaliste du développement économique soit celle venant d'en haut (top down). Les fonctionnaires de toute capitale étaient censés savoir ce qui était bon pour les régions les plus éloignées. N'avait-on pas en tête le modèle préconisé sous Roosevelt et faisant partie de son new deal : celui de la Tennessee Valley Authority. À la même époque, la France créait la Délégation à l'aménagement du territoire et à l'action régionale (DATAR $)^{6}$. On voulait reverdir le désert français en réponse au brulot de Jean-François Gravier ${ }^{7}$. Quant au Québec, suite à l'expérience du plan de développement du BAEQ, on espérait rien de moins en faire autant au Saguenay/Lac-Saint-Jean, dans le Nord-Ouest québécois, enfin partout où la nécessité se ferait sentir. Les enjeux politiques ayant eu préséance sur les enjeux économiques, la planification régionale fut reléguée aux oubliettes.

\section{La deuxième phase : les années 70}

Toujours inspiré par l'approche du développement par le haut, Montréal en vint à occuper le devant de la scène. $\mathrm{Vu}$ comme unique véritable pôle de croissance, comme l'écrit Bruno Jean dans ces pages, le rapport Martin-Raynault-Higgins invita à concentrer toute l'attention sur la métropole québécoise selon le principe : ce qui est bon pour Montréal est bon pour les régions. Mais, comme la politique ne peut se résigner à abandonner les régions à leur sort, on mit de l'avant des programmes visant l'implantation d'entreprises en retour d'avantages fiscaux. Le ministère fédéral de l'Expansion économique régionale tenta de mettre en pratique le concept de foot-lose enterprise, à savoir le recours à des entreprises indépendantes des ressources locales et des marchés approximatifs.
Ainsi, la Gaspésie faisait partie de ces «zones spéciales » où on espérait voir s'établir des entreprises n'ayant rien à voir avec les pêches, la forêt ou les mines. Cette fois ce sont les lois économiques qui feront perdre les illusions. Et la Gaspésie continuera de connaitre un solde migratoire négatif.

\section{La troisième phase : les années quatre-vingt à nos jours}

En 1982, le gouvernement du Québec publie le rapport Gendron Le choix des régions qui invite les forces vives régionales à occuper la place qui leur revient en ayant voix au chapitre. Trois ans plus tard, avec À l'heure de l'entreprise locale, Québec veut rapprocher le « haut » et le «bas » en profitant de la mise en fonction quelques années plus tôt des Municipalités régionales de comté. Conçues en 1978 en vue essentiellement de procéder à l'aménagement du territoire (où faire passer telle bretelle d'autoroute, quelles berges de rivières aménager, etc.) on pensa à en faire des espaces d'initiatives économiques. Ce faisant, l'État admettait ainsi ne plus pouvoir assumer seul le développement des régions. En 1992, sous l'instigation du ministre Yvon Picotte, une réforme mise de l'avant prendra le nom de «nouvelle politique régionale» et consistera à inciter les régions à prendre une plus grande part de responsabilité dans leur développement. La voie fut ainsi pavée en vue de la création, en 1997, des Centres locaux de développement qui seront rattachées aux MRC lesquelles auront à se frotter à la planification stratégique. Et, on en arrivera à parler de développement local défini par les officines gouvernementales comme étant le lieu de la mise en action des initiatives individuelles ou collectives en fonction des ressources sur un territoire précis. Ce fut la consécration du développement par le bas (bottomup). Restait à apprendre à travers l'expérience. Beaucoup demeurait à faire. Sur les origines et les formes prises par le développement local voir Simard, Diallo et Tremblay ${ }^{8}$.

\section{La voie fut ainsi pavée en vue de la création, en 1997, des Centres locaux de développement qui seront rattachées aux MRC lesquelles auront à se frotter à la planification stratégique.}




\section{UNE LEVÉE DE BOUCLIERS}

Le soir du 28 avril dernier, en recourant à la formule du bâillon, la loi 28 impliquant 337 articles a été adoptée. C'est ainsi que les Centres locaux de développement et les Conférence régionales des élus (CRÉ) sont passées à la trappe. Le gouvernement a donc fait peu de cas du mémoire du CRDT déposé à la Commission des finances publiques le 10 février qui soulignait les conséquences importantes du projet de loi 28 sur les institutions locales et régionales et le devenir de nos territoires ${ }^{9}$. On sait qu'en vertu du pacte fiscal provisoire signé par le monde municipal et l'État du Québec, à l'automne 2014, le gouvernement se proposait de réduire de 300 millions de dollars ses transferts aux municipalités, soit $10 \%$ du total des transferts. Quant aux montants destinés aux CLD, ils devaient être diminués de $55 \%$, soit de 40 millions de dollars. Il en reviendrait aux MRC de prendre la responsabilité du développement local et du soutien à l'entrepreneuriat. L'avenir des CLD dépendra désormais de la décision des élus de chaque MRC.

En fait, dès l'annonce du projet de loi 28 à l'automne dernier les réactions n'ont pas tardé et pas seulement de la part de l'opposition officielle. Nulle autre que Nathalie Normandeau, cette ancienne vice-première ministre du gouvernement Charest qui fut la pasionaria des régions n'a pu s'empêcher de dire :

«... le gouvernement n'a aucune vision en matière de développement régional et rural. Je suis très sévère, mais je trouve ça pitoyable, la façon dont ça se passe actuellement. On a une méconnaissance profonde des réalités régionales et rurales. »

Pour sa part, Mario Carrier, professeur de développement régional à l'Université Laval, a affirmé : «...les décisions du gouvernement viennent de changer l'équilibre du rapport de forces entre le monde rural et le monde urbain ». Il prévoit une minorisation de plus en plus affirmée du monde rural ${ }^{10}$. Mais, il fallait s'y attendre, ce sont les représentants des milieux concernés qui sont montés au créneau en créant le mouvement Touche pas à ma région ${ }^{11}$. Les premières lignes d'un manifeste présenté en janvier dernier se lisent ainsi :

« Nous, citoyens des régions du Québec, fils et filles de bâtisseurs, gens de cœur et de courage, revendiquons le droit de poursuivre le développement de nos régions selon les principes auxquels nous croyons. Par une gouvernance décentralisée et à notre image $»^{12}$.

La prise de position se poursuit par l'évocation d'une mobilisation de tout ce qui compose les forces vives des différents territoires du Québec qui refusent un mode de gouvernance au sein duquel on les prive d'une part importante de leur pouvoir décisionnel.

Pour la Gaspésie, le Conseil de la culture de la région fut le premier organisme à réagir contre les coupures annoncées concernant les CLD et les CRÉ. On met en garde contre des décisions susceptibles d'accentuer l'isolement de la région. Or, on signale qu'au travers les années une synergie a pu se développer entre les différents acteurs du développement régional et les instances gouvernementales, et ce, précisément grâce aux organismes mis en place par les différentes administrations gouvernementales. C'est ce que compromet par les décisions annoncées par l'administration Couillard ${ }^{13}$. Fautil rappeler que certains en arrivaient, dans les années 70, à se demander s'il ne valait pas mieux «fermer» la Gaspésie, en la rendant à l'état qu'avait pu connaître Jacques Cartier. Si on pouvait imaginer le reboisement et le retour possible du poisson, il en allait autrement pour les Indiens et les richesses du sous-sol.

La prise de position se poursuit par l'évocation d'une mobilisation de tout ce qui compose les forces vives des différents territoires du Québec qui refusent un mode de gouvernance au sein duquel on les prive d'une part importante de leur pouvoir décisionnel. 


\section{QUEL ESPOIR POUR LA GASPÉSIE?}

C'est la question que j'ai soulevée dans mon ouvrage paru en $2002^{14}$. Douze ans plus tôt, à l'invitation de trois jeunes agents de développement local, j'ai animé à Chandler une rencontre en présence d'une soixantaine de personnes identifiées aux parties prenantes de la région. Il s'agissait de favoriser une réflexion sur les conséquences éventuelles de la menace de la mise à pied du tiers des effectifs de la papetière Gaspésia. On connaît la suite. En décembre 2001, malgré le passage du premier ministre accompagné du quart du conseil des ministres, l'implication de divers partenaires et l'injection de 564 millions \$ en vue de transformer radicalement l'usine, ce ne fut pas suffisant. Moins de deux ans plus tard, la Gaspésia ferma ses portes. Sans vouloir jouer le rôle de rabat-joie, j'ai écrit que les Gaspésiens ne devaient pas attendre le renouvèlement d'un tel «cadeau » de la part du gouvernement. Je voyais l'avenir de l'ensemble de la région comme étant fonction de sa diversification économique grâce à l'entrepreneuriat local. Et à l'instar de ce qui s'observait dans d'autres régions depuis les années 90, la région devait miser sur la PME innovante à forte valeur ajoutée. Comment y parvenir?

\section{Il fallait donc prendre l'exemple de la région voisine le Bas-Saint-Laurent qui, au tournant du siècle, démontrait une étonnante vitalité en matière de PME innovantes.}

Il fallait d'abord cesser de voir la Gaspésie comme une région ressource étant donné que ses ressources se trouvaient en voie de disparition et que l'économie du XXI siècle n'en aurait de toute façon guère besoin. Il fallait donc prendre l'exemple de la région voisine le Bas-Saint-Laurent qui, au tournant du siècle, démontrait une étonnante vitalité en matière de PME innovantes. On avait une preuve éloquente que l'entrepreneuriat local était davantage qu'un slogan trop galvaudé. Bien sûr, les entrepreneurs en potentiel doivent disposer d'un accompagnement adéquat et ne pas se voir laisser à eux-mêmes. Que ce soit pour la prospection de nouveaux marchés, pour l'adoption à de nouvelles technologies, pour le recrutement et la formation de la main-d'œuvre, pour la prospection d'alliances stratégiques, l'information qui s'y rattache doit être fournie aux porteurs de projets. C'est (ou c'était...) la responsabilité des CLD que la loi 28 pourrait faire disparaitre. À l'instar de leurs consœurs relevant du fédéral, les Sociétés d'aide au développement des collectivités (SADC), les CLD de chacune des MRC gaspésiennes ont montré leur capacité de s'insérer dans une démarche favorisant la rencontre entre l'ascendant (bottom-up) et le descendant (top down) en vue de cette indispensable diversification. C'est ce que montre une étude récente de l'INRPME de l'UQTR.

On précise que la Gaspésie se caractérise par des activités reliées à l'agroalimentaire, aux ressources marines et, bien sûr, au tourisme. Les PME faisant partie de l'échantillon étudié se spécialisent dans la transformation des produits de la mer et font ainsi partie du réseau d'excellence de la région. En affichant un taux d'innovation de $45 \%$, ces PME ne s'éloignent guère des taux observés en régions situées près de Montréal. Les entrepreneurs locaux savent que leur sort dépend de l'innovation. C'est ce qui permet d'exporter aux États-Unis et en Asie. Est-ce nécessaire de préciser que ces PME font appel aux services de différents organismes intérieurs tels les CLD et extra-régionaux pour être à la fine pointe du progrès afin de répondre aux exigences du marché ${ }^{15}$. L'exemple suivant de la SODIM présenté lors de l'URQ 2011 illustre bien l'importance de l'accompagnement dont les entreprises locales peuvent bénéficier.

La société de développement de l'industrie maricole inc. (SODIM), est une société à but non lucratif créée en 1997 dont l'avenir se trouve compromis par la politique d'austérité du gouvernement. Elle a pour mission de favoriser le développement d'une industrie maricole viable sur le territoire de la corporation, soit en Gaspésie, aux Îles-de-laMadeleine, au Bas-Saint-Laurent et en Côte-Nord, notamment en : offrant une aide financière aux entreprises de la filière, des services d'aide technique et en favorisant la recherche et développement et le transfert technologique ${ }^{16}$. Elle intervient auprès de Pêcherie Allard inc. dont le dirigeant donna, lors de l'Université rurale québécoise 2011, une vue d'ensemble des défis passés et actuels de son entreprise. Cette PME se voulait innovante en tant que pionnière dans le 
domaine de l'élevage des moules en milieu dit « ouvert» (recours aux boudins en continu). Comme l'a attesté le responsable de la SODIM, le succès d'une telle entreprise exige une volonté régionale et gouvernementale et un arrimage étroit avec les organismes locaux ${ }^{17}$.

La tenue de 1'URQ 2011 dans la Baie-desChaleurs/Les Plateaux a coïncidé avec une donnée démographique tout à fait inusitée: pour la

\section{CONCLUSION}

Ce dynamisme saura-t-il se poursuivre en dépit des nombreuses coupures décrétées par la présente administration Couillard? Le scepticisme est de mise quand on sait Solidarité rurale, créé en 1991, avec pour responsabilité d'être le principal intermédiaire dans la mise en œuvre des politiques rurales a été forcé de disparaître. En ira-t-il de même pour l'Université rurale québécoise, créée en 1997, dont la dixième édition en 2015 dans la région de Charlevoix a été annulée?

Dans mon ouvrage de 2002, j'écris :

«Oui, les forces vives des milieux locaux ont beaucoup appris et continuent d'apprendre. Il n'est pas exagéré de signaler qu'en matière de stratégies de développement local dix ou quinze années constituent un horizon bien court. Le Québec fait actuellement ses classes à l'aide des structures implantées sur l'ensemble de son territoire (p. 140) ».

\section{Qu'adviendra-t-il de cette expérience de « travailler ensemble » acquise à travers les années?}

Les CLD font partie de ces structures comme d'autres que l'actuel gouvernement a fait disparaître. Or, ces 13 dernières années, tout un chacun à l'échelon local avait effectivement beaucoup appris. Qu'adviendra-t-il de cette expérience de «travailler ensemble» acquise à travers les années? C'est la question soulevée dans le mémoire présenté par l'Abitibi-Témiscamingue :

«Avec ses récentes décisions de démanteler des structures de développement que nous avons mises en place, et cela sans ouverture première fois «de mémoire d'homme» la Gaspésie a affiché en 2010 un solde migratoire positif. On comprend l'enthousiasme affiché par les organisateurs de l'URQ qui, dans leur rapport, font allusion au dynamisme de la région et à la passion qui anime ses habitants. L'innovation ayant été le thème dominant de cette rencontre biennale, il fut possible de démontrer que les Gaspésiens savaient relever le défi de la diversification économique ${ }^{18}$.

au dialogue, le gouvernement du Québec brise l'élan que nous nous sommes donné. Il nous prive également de leviers de développement essentiels à notre épanouissement, tant social qu'économique. ${ }^{19}{ }$ »

Les forces vives en région devront faire preuve de résilience et de vigilance en évitant de perdre les acquis des quelque trente dernières années. Ne pas jeter l'éponge avec l'espoir que le bon sens saura l'emporter et que le gouvernement, fidèle à la position adoptée en 1992, poursuivra son travail d'accompagnateur pour le mieux-être de nos régions. Si des villages de moins de 500 habitants ne pourront éviter de disparaître, à tous le moins nos régions périphériques conserveront leur place au soleil.

\section{BIBLIOGRAPHIE ET NOTES}

${ }^{1}$ http://www.touchepasamaregionbsl.org/

${ }^{2}$ Émision du 12 avril 2015.

${ }^{3}$ Le développement agricole avec référence à la situation de l'Est québécois, Mémoire présenté en vue de l'obtention du grade de maître en sciences économiques, Université catholique de Louvain, 1968.

${ }^{4} \mathrm{~K}$. Lévesque et $\mathrm{K}$. Gagnon, Le portait de la dévitalisation, La Presse, 10 avril 2014.

${ }^{5}$ Le président du Conseil du patronat n'a pas tardé à nuancer sa prise de position suite aux réactions engendrées.

${ }^{6}$ Dont le siège se trouvait à la proximité de la Tour Eiffel Signe des temps : l'édifice a été vendu à des intérêts étrangers il y a peu de temps.

${ }^{7}$ Paris et le désert français, Paris, Le Portulan, 1947.

${ }^{8}$ Simard, M, Diallo, T, et Tremblay, S. (2015) Théories et pratiques en développement territorial : des grands projets aux initiatives locales et vice versa, Organisations \& Territoires, vol 23, no 3, pp 5-14. 
${ }^{9}$ Centre de recherche en développement territorial, Mémoire présenté à la Commission des Finances publiques, 10 février 2015.

${ }^{10} \mathrm{~K}$.Lévesque et K. Gagnon op.cit.

${ }^{11}$ Un pastiche du slogan français des années 80 : Touche pas à mon pote!

${ }^{12} \mathrm{http}: / /$ touchepasamesregions.ca/janvier2015

${ }^{13} \mathrm{http} / / / \mathrm{www}$.culturegaspesie.org/fr/ActualitesDetails.as px?Id=1201

${ }^{14}$ Le développement local: comment stimuler l'économie des régions en difficulté, Québec, Les Éditions de l'IRQ/PUL.

${ }^{15}$ INRPME, 2014, Influence de la culture entrepreneuriale régionale sur l'innovation des PME : analyse de deux régions contrastées du Québec, Université du Québec à Trois-Rivières.

${ }^{16} \mathrm{http}: / /$ www.sodim.org/mission.htm

${ }^{17} \mathrm{http} / / / \mathrm{www}$. uqar.ca/files/urq/2011/e2_pm-

presentation_sylvain-lafrance_urq_110907.pdf.

L'entreprise a cessé ses activités en 2013.

${ }^{18} \mathrm{http} / / / \mathrm{www} . u q a r . c a / f i l e s / u r q / 2011 /$ conclusions_et_per spectives.pdf

${ }^{19} \mathrm{http}: / /$ mobilisation-abitibi-temiscamingue.org/ 